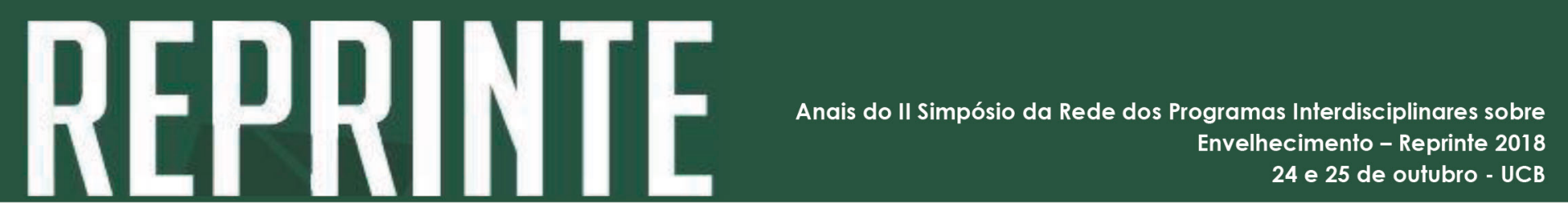

http://dx.doi.org/10.5335/rbceh.v16i1.9920

\title{
29) Uso de soluções e-Health para o monitoramento das condições de saúde de pacientes hipertensos
}

\author{
Raquel Debon' ${ }^{1}$ Simiane Volpi ${ }^{1}$; Ana Carolina Bertoletti De Marchi²
}

\section{Resumo}

A hipertensão está cada vez mais presente na população mundial, principalmente em idosos, gerando onerosos custos a saúde pública. Nesse cenário as aplicações e-Health através de tecnologias computacionais, buscam melhorar as condições de saúde de pacientes por meio do monitoramento dos níveis pressóricos. Diferentes estudos vem sendo realizados com essas aplicações, trazendo resultados positivos e melhoras significativas nos níveis de pressão arterial. As soluções e-Health trazem melhoras nas qualidade de saúde de pacientes hipertensos mas são necessários maiores estudos para validar sua eficácia.

Palavras-chave: hipertensão, e-Health, saúde pública.

\section{Introdução}

A Organização Mundial da Saúde estima que cerca de 600 milhões de pessoas tenham Hipertensão Arterial Sistêmica (HAS) e anualmente 7,1 milhões vão a óbito, gerando onerosos custos aos sistemas de saúde (MALTA et al, 2017). As soluções e-Health utilizam tecnologias computacionais para auxiliar nos cuidados com a saúde e se tornaram uma ferramenta importante para o monitoramento das condições de saúde (WANG et al, 2014).

\section{Objetivo}

Analisar as soluções e-Health para o monitoramento de pacientes hipertensos.

1 Mestrandas no Programa de Pós-Graduação em Envelhecimento Humano, Universidade de Passo Fundo. Endereço para correspondência: BR 285, São José, CEP 99052-900 - Passo Fundo, Brasil. Emails (na ordem em que aparecem): raquedebon@hotmail.com; simivolpi@yahoo.com.br

2 Professora do Programa de Pós-Graduação em Envelhecimento Humano, Universidade de Passo Fundo. carolina@ upf.br 


\section{Métodos}

Trata-se de uma revisão de literatura realizada nas bases de dados BVS e Science, no mês de setembro de 2018. Resultados: Foram analisados 10 estudos. A adesão da tecnologia em prol da saúde vem ganhando cada vez mais adeptos para a promoção, prevenção e qualidade de vida. Diferentes estudos vêm sendo realizados a partir do desenvolvimento de aplicativos móveis (app) para pacientes hipertensos. Em sua grande maioria, fazem uso de um banco de dados em que o usuário alimenta o sistema com aferições da pressão arterial semanalmente ou quinzenalmente, por um período mínimo de 15 dias e, no máximo, de seis meses. Além disso, os pacientes recebem alertas e feedback por parte dos profissionais de saúde, o que estimula a continuidade do uso da solução móvel. O app BMAP foi desenvolvido para hipertensos visando controlar a doença através de mudanças nas condições de saúde (ASHOORKHANI et al, 2016). De forma semelhante, Albini et al (2016) desenvolveram um app que visa o controle da HAS.

\section{Conclusão}

É possível perceber que as aplicações e-Health contribuem de forma positiva na melhoria da qualidade de vida. Contudo, ainda são necessários maiores estudos para verificar a validade de soluções e-Health com a população brasileira hipertensa.

\section{Referências}

ALBINI, F. et al. An ICT and mobile health integrated approach to optimize patients' education on hypertension and its management by physicians: The Patients Optimal Strategy of Treatment(POST) pilot study. 2016 38th Annual International Conference of the IEEE Engineering in Medicine and Biology Society (EMBC). Anais...IEEE, ago. 2016.

ASHOORKHANI, M. et al. Comparing the effectiveness of the BPMAP (Blood Pressure Management Application) and usual care in self-management of primary hypertension and adherence to treatment in patients aged 30-60 years: study protocol for a randomized controlled trial. Trials, v. 17, n. 1, p. 511, dez. 2016.

MALTA, D. C. et al. Prevalence of and factors associated with self-reported high blood pressure in Brazilian adults. Revista de Saúde Pública, v. 51, n. suppl 1, 2017.

WANG, J. et al. Smartphone Interventions for Long-Term Health Management of Chronic Diseases: An Integrative Review. Telemedicine and e-Health, v. 20, n. 6, p. 570-583, 3 jun. 2014. 